\title{
O PAPEL MODERADOR DA EDUCAÇÃO EMPREENDEDORA DIANTE DA INTENÇÃO EMPREENDEDORA
}

\section{THE MODERATING ROLE OF ENTREPRENEURIAL EDUCATION IN ENTREPRENEURIAL INTENTION}

\author{
Cristiane Krüger \\ Universidade Federal de Santa Maria - UFSM RS \\ Rafaela Escobar Bürger \\ Universidade Federal de Santa Catarina - UFSC \\ Italo Fernando Minello \\ Universidade Federal de Santa Maria - UFSM RS
}

Submissão: $10 / 08 / 2018$

Aprovação: 04/06/2019

\begin{abstract}
RESUMO
O comportamento empreendedor é uma proposta de solução para o mercado de trabalho em crise, especialmente nos períodos de mudança econômica e social. Com base na Teoria do Comportamento Planejado (TCP), este estudo analisa a relação entre conhecimento empreendedor e experiência prévia diante da intenção e educação empreendedoras. Testes estatísticos foram utilizados para analisar as respostas de uma amostra de 401 indivíduos que responderam o Questionário de Intenção Empreendedora, de Liñan e Chen (2009). Os resultados mostraram que indivíduos com conhecimento empreendedor e experiência prévia apresentaram pontuações superiores diante de indivíduos sem o respectivo conhecimento e experiência. Esses achados contribuem para o entendimento da intenção empreendedora em indivíduos com conhecimento empreendedor e experiência, consolidando o uso do modelo TCP para estudar a intenção empreendedora individual. Os resultados sugerem que os atores responsáveis devem prestar atenção na promoção da educação empreendedora, contemplando conhecimento e experiência prévia, se estiverem interessados em promover o comportamento empreendedor.
\end{abstract}

Palavras-chave: empreendedorismo, intenção empreendedora, educação empreendedora, experiência prévia. 


\begin{abstract}
Entrepreneurial behavior is a proposed solution to the labor market in crisis, especially in periods of economic and social change, in that sense, politicians look for ways to promote it. Based on the theory of planned behavior (TCP), this study analyzes the relationship between entrepreneurial knowledge and previous experience with entrepreneurial intention and education. Statistical tests were used to analyze the responses of a sample of 401 individuals who answered the Questionnaire Entrepreneurial Intent, by Liñan and Chen (2009). The results showed that individuals with entrepreneurial knowledge and previous experience presented higher scores in front of individuals without their respective knowledge and experience. These findings contribute to the understanding of the entrepreneurial intention in individuals with entrepreneurial knowledge and experience, consolidating the use of the TCP model to study the individual entrepreneurial intention. The results suggest that politicians should pay attention to the promotion of entrepreneurial education, contemplating knowledge and previous experience, if they are interested in promoting entrepreneurial behavior.
\end{abstract}

Keywords: Entrepreneurship, entrepreneurial intention, entrepreneurial education, previous experience. 


\section{INTRODUÇÃO}

Durante as últimas décadas, o desenvolvimento do espírito empreendedor tem sido colocado, não apenas no Brasil, mas em diversos países do mundo, como prioritário nas agendas e debates políticos, econômicos e acadêmicos, devido a comprovada influência que $o$ mesmo exerce no desenvolvimento econômico e social de um país (PAUL; SHRIVATAVA, 2016). Colaborando com isso, muitos estudos empíricos apontam que o comportamento empreendedor, ou pelo menos alguns aspectos dele, podem ser ensinados e que a educação pode ser considerada um dos instrumentos chave para promover atitudes, intenções e competências empreendedoras (KURATKO, 2016; MCNALLY et al., 2013). Nesse sentido, aponta-se a educação empreendedora como uma das formas mais eficientes de se estimular a formação de novos empreendedores (ROCHA; FREITAS, 2014).

Lima et al. (2015) apontam que o interesse pela educação empreendedora cresceu significativamente na última década e várias razões são apontadas para esse fato. Dentre elas, destaca-se a constatação de que a formação empreendedora contribui para o surgimento de novas empresas, para a criação de novos postos de trabalho e para o desenvolvimento da inovação nas organizações em geral (GUERRA; GRAZZOTIN, 2010; LANERO et al., 2011; LIMA et al., 2015).

A partir de um relatório, a Comissão Europeia (2007) reforça a relevância de programas de formação empreendedora identificando países como Espanha, Irlanda, Chipre, Polônia e Reino Unido que contemplam o empreendedorismo nos currículos de ensino básico. Além disso, destaca-se que a referida Comissão, em 2012, estabeleceu diretrizes estratégicas para o avanço do perfil empreendedor através da educação como uma das principais ações para a promoção da atividade empreendedora no bloco econômico europeu (COMISSÃO EUROPEIA, 2012).

Apesar da unanimidade a respeito da importância de desenvolver o comportamento empreendedor por meio da educação empreendedora, ainda não há homogeneidade nas discussões acadêmicas a respeito do que determina a eficiência dessa educação. Maresch, Kailer e Wimmer-Wurm (2016) apontam que na literatura, percebe-se concordância de que os modelos de educação empreendedora são baseados nas teorias de intenção empreendedora a qual, segundo Kuehn (2008), precede o comportamento empreendedor, podendo assim, beneficiar os indivíduos que discutem o desenvolvimento do empreendedorismo.

$\mathrm{Na}$ tentativa de compilar resultados de estudos desta natureza, recentes análises foram conduzidas e comprovaram a existência de uma relação positiva entre a educação empreendedora e a intenção empreendedora (BAE et al., 2014; MARTIN et al., 2013).

Nesse sentido, o presente estudo segue a tradição de pesquisa empreendedora baseada em evidências, trazendo como contribuição acadêmica a discussão sobre a educação empreendedora e a intenção empreendedora de alunos de uma instituição de ensino superior. Diante desse panorama e da evolução dos estudos sobre educação empreendedora, o presente estudo tem como objetivo analisar a intenção empreendedora de alunos de graduação de uma universidade diante da perspectiva da educação empreendedora. Em termos específicos, almeja-se: i) avaliar a intenção empreendedora dos alunos de graduação e ii) identificar o conhecimento em empreendedorismo e as experiências profissionais dos alunos iii) verificar a relação entre o conhecimento em empreendedorismo, experiências profissionais e a intenção empreendedora.

\section{EDUCAÇÃO EMPREENDEDORA}


Educação empreendedora para Cope (2005), pode ser entendida como um processo dinâmico de conscientização, associação e aplicação que envolve transformar a experiência e o conhecimento em resultados aprendidos e funcionais. Para o autor educação empreendedora compreende ainda conhecimento, comportamento e aprendizagem efetivo-emocional (COPE, 2005).

Visto que os empreendedores não são nascidos e sim desenvolvidos, torna-se necessário incluir nos currículos o ensino do empreendedorismo (PINTO, 2013). Para Lopes (2010, p. 165) "o conceito de educação empreendedora a ser adotado deve ser muito mais no sentido de viabilização de propósitos do que apenas no sentido de abertura de um negócio.". Esse conceito envolve e direciona o presente estudo.

A ação é fundamental no processo de educação empreendedora, de acordo com Neck e Greene (2011), tem como primeiro passo permitir que os alunos experimentem a realidade, para depois aprenderem conceitos. Neste sentido pondera-se que o papel das instituições de ensino, as quais representam papel de suma importância para a concretização desse aprendizado empreendedor. Desenvolver o comportamento empreendedor no aluno é capacitá-lo para a criação, condução e implementação do processo criativo proporcionando a oportunidade de elaborar novos planos de vida, de trabalho, de estudo, de negócios, transformando-se, deste modo, no ator responsável pelo seu desenvolvimento pessoal, bem como de sua organização (SOUZA et al., 2006).

Logo, a educação empreendedora pode oportunizar ao estudante enxergar e avaliar determinada situação, assumindo uma posição proativa frente a ela, capacitando-o a elaborar e planejar formas e estratégias de interagir com aquilo que ele passou a perceber (LOPES, 2014). Além disso, essa educação auxilia na compreensão da realidade, estimulando a reflexão sobre transformações e inovações, buscando ações planejadas e tecnicamente embasadas, além de estimular a transformação positiva da sua realidade, nas esferas pessoal, econômica e social (SEBRAE, 2011).

Sob essa perspectiva, Singer, Amorós e Arreola (2015), salientam que o conteúdo empreendedor deve ser inserido nos três níveis de educação de forma sistemática e consistente, com vistas ao desenvolvimento de uma cultura empreendedora que permeie a sociedade como um todo. Dentro desse enfoque, destaca-se o papel da instituição de ensino superior que pode ser fomentadora do preparo para empreender, desenvolver novas formas de ensinar que despertem em seus alunos uma mentalidade empreendedora.

Nessa perspectiva, para se atingir os diferentes objetivos propostos pela educação empreendedora, necessita-se traçar um plano de ensino que se adapte a metodologia pedagógica ao contexto da aprendizagem esperada (ROCHA; FREITAS, 2014). Segundo Balconi (2016), atividades de ensino práticas são as mais propensas para contribuir para o estímulo do comportamento empreendedor. Entre as opções pedagógicas citam-se: palestras, recomendações de leituras, estudos de caso, visita a empresas, brainstorming, simulações e projetos desenvolvidos em grupos (DOLABELA, 2008).

Visto que o comportamento empreendedor pode ser aperfeiçoado mediante processo de aprendizagem (POLITIS, 2005; TSENG, 2012; ZAMPIER, TAKAHASHI, 2014), ressalta-se a importância da inserção da educação empreendedora nas instituições de ensino, as quais, segundo Pereira (2010), devem desempenhar um papel de agente promotor, disseminando uma cultura empreendedora em todos os níveis. A eficácia dessa educação, segundo Kuratko (2016) e Rauch e Hulsink (2015), está diretamente ligada à fatores que impulsionam a intenção empreendedora. Esta, por sua vez, segundo avaliação meta-analítica desenvolvida por Schlaegel e Koenig, (2014) sugere que os fatores que impulsionam a 
intenção empreendedora são atitudes, normas subjetivas e controle do comportamento percebido, elementos oriundos da TCP de Ajzen (1991).

\section{INTENÇÃO EMPREENDEDORA}

Segundo Tubbs e Ekerberg (1991), as intenções ocupam posição privilegiada no estudo do comportamento humano. Para os autores, intenção representa um objetivo que um indivíduo almeja alcançar e também, o planejamento que fundamentará a busca pela realização do objetivo. A intenção de um indivíduo intrinsecamente ligada ao seu comportamento, ou seja, todo o comportamento é precedido de uma intenção (KRUEGER et al., 2000). A partir disso, a intenção empreendedora pode ser definida como a "convicção auto-reconhecida por uma pessoa de que pretendem criar um novo empreendimento e conscientemente planejam fazê-lo em algum momento no futuro" (THOMPSON, 2009, p. 667).

Para Thompson (2009), intenção empreendedora individual é um constructo chave na investigação sobre a formação de novos negócios. Mais recentemente, um estudo longitudinal de Kautonen et al. (2015) confirmou que a intenção empreendedora prevê ação empresarial. Assim, a questão do que influencia a intenção empreendedora é relevante para os formuladores de políticas, profissionais e educadores. Nesse sentido, a intenção empreendedora tornou-se um campo vibrante na pesquisa em empreendedorismo (FAYOLLE; LINAN, 2014), uma vez que "as intenções têm provado ser o melhor preditor do comportamento planejado, particularmente quando esse comportamento é raro, difícil de observar ou envolve atrasos imprevisíveis" (KRUEGER et al., 2000, p. 411).

Diante dessa perspectiva, Liñán e Chen (2009) propuseram um modelo de mensuração da intenção empreendedora adaptado da Teoria do Comportamento Planejado (TCP) de Ajzen (1991), conforme exposto na Figura 1.

Figura 1- Modelo da Intenção Empreendedora (QIE)

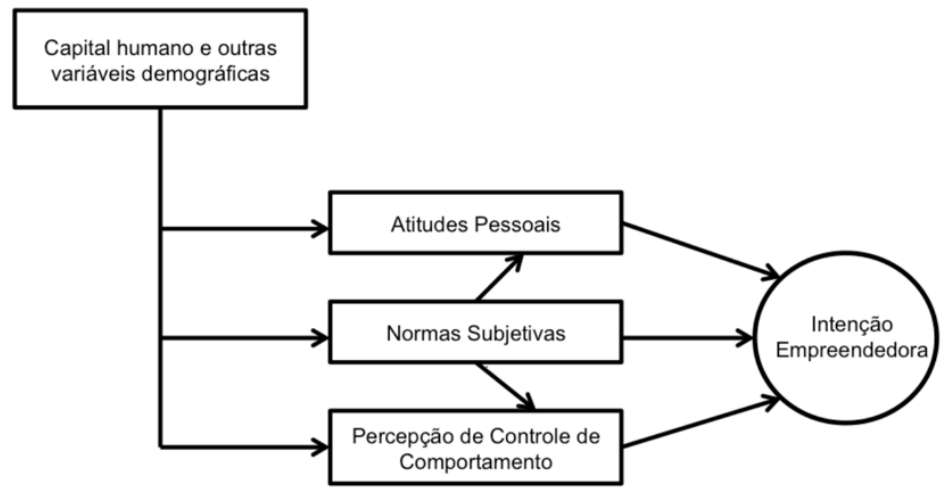

Fonte: LIÑÁN; CHEN, 2009, p. 597.

Conforme exposto, a intenção empreendedora baseia-se, primeiramente, na TCP (AJZEN, 1991), que, para os autores Schlaegel e Koenig (2014) e Krueger e Carsrud (1993), fornece uma base teórica sólida. Os autores postulam que o comportamento futuro de uma pessoa é precedido por intenção: quanto mais forte a intenção de uma pessoa se engajar em 
um comportamento específico, mais provável é que o comportamento real será realizado. Além disso, a intenção de realizar um determinado comportamento é o resultado de três antecedentes cognitivos: (i) atitude em relação ao comportamento; (ii) normas subjetivas; e (iii) controle comportamental percebido (SCHLAEGEL; KOENIG, 2014; KRUEGER; CARSRUD, 1993).

A atitude frente ao comportamento refere-se à avaliação do individuo a respeito do seu próprio comportamento, seja esta positiva ou negativa (OLIVEIRA et al., 2016). A avaliação é o componente mais afetivo da atitude, determinando a motivação e a força da intenção do comportamento. Nesse sentido, a atitude favorável está associada a uma maior intenção de agir (MORIANO; PALACÍ; MORALES, 2007).

As normas subjetivas dizem respeito à pressão social exercida para realizar - ou não um comportamento e reflete o efeito dos valores sociais sobre o indivíduo (MORALES; REBOLLOSO; MOYA, 1994). A norma subjetiva é a componente mais social do modelo, na medida em que incorpora a influência de pessoas significativas para o sujeito na decisão de desenvolver a sua carreira profissional por meio do empreendedorismo (OLIVEIRA et al., 2016).

Em relação ao controle comportamental percebido, as pessoas manifestam comportamentos que acham que são capazes de controlar e dominar (BANDURA, 1982). Esse conceito consiste na capacidade para manifestar um comportamento, por exemplo, iniciar uma atividade empresarial (OLIVEIRA et al., 2016). Segundo Baron (2002), a literatura traz a auto eficácia como uma característica do perfil, em função disso, aponta-se que indivíduos com um nível de auto eficácia elevado sejam mais persistentes, trabalhem de forma mais efetiva, se esforcem mais na realização das tarefas e tenham a confiança de possuir as competências necessárias para conseguir os seus objetivos.

\section{Hipóteses da pesquisa}

Uma vez definido o objetivo do estudo, foram estabelecidas as seguintes hipóteses iniciais de trabalho representativas, das variáveis do modelo, dos antecedentes pessoais, dos conhecimentos empreendedores, da inter-relação entre as variáveis do modelo e da comparação da intenção empreendedora. Deste modo, estabeleceram-se as seguintes hipóteses (Figura 2).

Figura 2 - Hipóteses da pesquisa 
Atitudes Pessoais

Normas Subjetivas

Percepção de

Controle de

Comportamento
H1

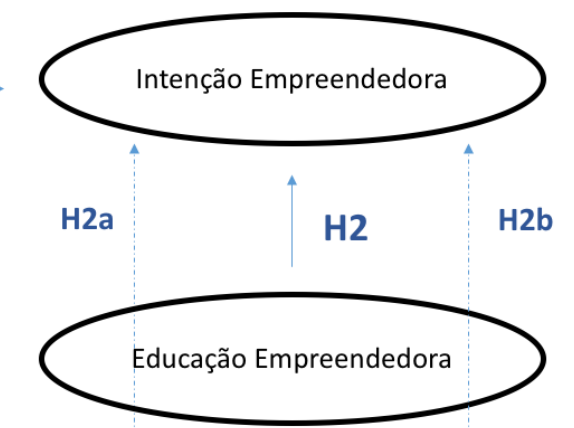

Conhecimento em empreendedorismo

Experiência profissional

Fonte: os autores.

Hipóteses:

$\mathrm{H}_{1}$. Existe uma relação positiva entre (1) atitudes pessoais, (2) normas subjetivas e (3) percepção de controle percebido, e a intenção empreendedora.

H2. Existe relação positiva entre educação empreendedora e intenção empreendedora.

$\mathrm{H}_{2 \mathrm{a}}$. Existe relação positiva entre conhecimento empreendedor e níveis mais elevados de intenção empreendedora.

$\mathrm{H}_{2 b}$. Existe relação positiva entre experiência profissional e níveis mais elevados de intenção empreendedora.

\section{METODOLOGIA}

A abordagem utilizada na presente pesquisa é quantitativa, descritiva e exploratória, baseada em pesquisa teórico-empírica. Para Ramos (2013) a utilização de métodos quantitativos para análise da realidade social presente em um mesmo estudo ou separados em estudos diferentes, tem o propósito de descrever e/ou comparar características de grupos sociais, realidades, contextos ou instituições e; estabelecer possíveis relações. A pesquisa do tipo descritiva procura descrever as características de uma determinada população ou os fatos e fenômenos de uma realidade, o que pode proporcionar uma maior familiaridade com o problema, tornando-o mais explícito e favorecendo o aprimoramento de ideias e considerações dos mais variados aspectos ligados ao fato estudado (TRIVIÑOS, 1987). Neste sentido, o tipo de estudo exploratório é especialmente adequado, pois o pesquisador dispõe de poucas informações em área na qual há pouco conhecimento acumulado e sistematizado (VERGARA, 2012).

O instrumento utilizado foi o Questionário de Intenção Empreendedora (QIE) de 
Liñán e Chen (2009), validado no Brasil por Hecke (2011). Atualmente o QIE compõe o estudo GUESS (LIMA et al., 2014).

A população total desta pesquisa é composta por 1560 indivíduos do Centro de Ciências Naturais e Exatas (CCNE) da Universidade Federal de Santa Maria (UFSM). Para o cálculo do tamanho mínimo da amostra optou-se pela fórmula a seguir (Equação 1), que segundo Fonseca e Martins (1996) é para uma população finita, considerou-se um nível de confiança de $95 \%$ e um erro padrão de 5\%, a amostra mínima calculada foi de 309 indivíduos.

Sendo:

$$
n=\frac{z_{\alpha / 2}^{2} \cdot p \cdot q \cdot N}{e^{2}(N-1)+Z_{\frac{\alpha}{2}}^{2} \cdot p \cdot q}
$$

e - erro amostral;

$\mathrm{N}$ - tamanho da população;

$\mathrm{n}$ - amostra mínima

$\langle=0,05-$ Nível de Significância

$\mathrm{p}=0,5$ e $\mathrm{q}=0,5$

$Z_{\frac{\alpha}{2}}^{2}=1,96$ para um nível de confiança de $95 \%$.

A coleta de dados ocorreu nos meses de novembro a dezembro de 2015, por meio de entrega pessoal nas salas de aula. Participaram 407 indivíduos, destes, foram desconsiderados 6 instrumentos preenchidos de modo incompleto, os respondentes superaram a amostra mínima estipulada.

Os dados foram tabulados e codificados, e, posteriormente conferidos, objetivando verificar possíveis erros de digitação. Para tratamento e análise dos dados coletados foram realizados testes estatísticos utilizando o software "Statistical Packagefor the Social Sciences" - SPSS, versão 24. Na ocasião foram analisados quantitativamente e explorados os dados do modelo proposto por Liñán e Chen (2009). A seguir apresenta-se a análise dos resultados.

\section{ANÁLISE DOS RESULTADOS}

$\mathrm{Na}$ análise dos resultados são identificadas as características da amostra e em seguida os demais resultados e discussões.

\subsection{CARACTERÍSTICAS DA AMOSTRA}

A amostra é composta por 401 indivíduos, sendo 182 pessoas do gênero masculino (45\%) e 219 do gênero feminino (55\%). No que se refere a faixa etária $34 \%$ dos pesquisados possuem menos de 19 anos, $41 \%$ possuem idade entre 20 e 23 anos, $25 \%$ dos pesquisados possuem mais de 24 anos. Em relação ao estado civil, os solteiros representam $89 \%$ dos respondentes (358 alunos). Quanto a experiência prévia, 71\% dos respondentes (283 alunos) afirmam que não possuem experiência profissional. Quanto ao conhecimento empreendedor, 91\% dos respondentes (364 alunos) afirmam nunca ter cursado quaisquer disciplinas relacionadas ao empreendedorismo.

\subsection{RESULTADOS E DISCUSSÕES}


Inicialmente foi realizado o teste de normalidade Kolmogorov-Smirnov e os resultados são expostos no Quadro 1.

Quadro 1 - Teste de Normalidade Kolmogorov-Smirnov

\begin{tabular}{|l|c|c|c|c|}
\hline \multicolumn{1}{|c|}{ Dimensões } & Estatística & gl & Sig. & Skewness \\
\hline Atitude pessoal &, 084 & 401 &, 000 & $-0,380$ \\
\hline Normas subjetivas &, 117 & 401 &, 000 & $-0,476$ \\
\hline Percepção de controle &, 081 & 401 &, 000 & 0,228 \\
\hline Intenção Empreendedora &, 095 & 401 &, 000 & 0,334 \\
\hline
\end{tabular}

Fonte: elaborado pelos autores.

A partir do Quadro 1 infere-se que os escores de atitude pessoal, normas subjetivas, percepção de controle e intenção empreendedora indicam que a distribuição dos dados difere significativamente de uma distribuição normal (Sig. < 0,05). No entanto, para Leech, Barret e Morgan (2005) pode-se considerar as variáveis aptas para testes paramétricos quando o Skewness constar entre -1> e <1, quando ocorre uma normalidade aproximada permitindo que se apliquem testes paramétricos. Neste caso para as quatro dimensões do QIE realizou-se testes paramétricos.

Com relação à adequação da amostra foi utilizada a medida de Kaiser-Meyer-Olkin, para Hair Jr. et al (2010) valores aceitáveis estão entre 0,5 a 1,0, obteve-se um KMO de 0,912, considerado excelente (HAIR JR. et al., 2010), valor que indica que a análise fatorial é apropriada. Também foi realizado o teste de esfericidade de Bartlett com Sig. de 0,000, rejeitando-se a hipótese nula de que a matriz de correlação das variáveis é uma matriz identidade (HAIR JR. et al., 2010).

Para estimar a confiabilidade, precisão do instrumento, foi medida a consistência interna através do alfa de Cronbach (SAMPIERI; COLLADO; LUCIO, 2013). O alfa de Cronbach é calculado pela seguinte Equação 2:

$$
\alpha=\frac{k}{k-1}\left[\frac{\sigma_{\tau}^{2}-\Sigma_{i=1 \sigma_{i}^{2}}^{k}}{\sigma_{\tau}^{2}}\right]
$$

Onde:

$\mathrm{k}$ corresponde ao número de itens do questionário;

$\int^{\wedge} 2 \mathrm{i}$ corresponde soma das variâncias de cada item;

$\int^{\wedge} 2 \mathrm{t}$ corresponde a variância total do questionário, determinada como a soma de todas as variâncias.

O alfa de Cronbach para o QIE totalizou $\langle=925$, atestando fidedignidade para a medida e avaliação do construto. Em seguida foram calculados mínimo, máximo, média, desvio padrão, variância e o alfa de Cronbach de cada dimensão para o construto estudado (Quadro 2).

Quadro 2 - Estatística descritiva

\begin{tabular}{|l|c|c|c|c|c|c|c|}
\hline \multicolumn{1}{|c|}{ Dimensões } & $\begin{array}{c}\mathbf{n}^{\mathbf{0}} \\
\text { Itens }\end{array}$ & Mínimo & Máximo & Média & $\begin{array}{c}\text { Desvio } \\
\text { Padrão }\end{array}$ & Variância & $\begin{array}{c}\text { Alfa de } \\
\text { Cronbach }\end{array}$ \\
\hline Atitude pessoal & 5 & 5,00 & 25,00 & 16,379 & 4,8663 & 23,681 & 0,858 \\
\hline Normas subjetivas & 3 & 3,00 & 15,00 & 11,626 & 2,5475 & 6,490 & 0,815 \\
\hline Percepção de controle & 6 & 6,00 & 30,00 & 15,284 & 5,6510 & 31,934 & 0,878 \\
\hline \hline
\end{tabular}




\begin{tabular}{|l|l|l|l|l|l|l|l|}
\hline Intenção Empreendedora & 6 & 6,00 & 30,00 & 14,713 & 6,5673 & 43,130 & 0,907 \\
\hline
\end{tabular}

Fonte: elaborado pelos autores.

Constata-se que devido a variação da quantidade de itens entre as dimensões do respectivo construto, os valores mínimos e máximos seguem essa prerrogativa, tendo em vista que cada dimensão se dá por meio da soma de seus itens. Nesse sentido, o menor valor de desvio padrão ocorre em normas subjetivas, que apresenta três itens, da mesma forma como o menor valor de variância. Observa-se que percepção de controle e intenção empreendedora possuem seis itens cada, dentre estes a dimensão de intenção empreendedora apresentou maior desvio padrão e variância, quanto maior, mais esse valor está distante da média. Diante dos valores da média verifica-se que a dimensão intenção empreendedora obteve a menor média, ao comparar o respectivo valor com a pontuação máxima, de modo geral os discentes pesquisados apresentam as quatro dimensões do construto pesquisado. Quanto aos resultados do alfa de Cronbach, infere-se que, tanto os resultados do QIE quanto das respectivas dimensões ficaram próximos de 1,0 o que indica uma maior confiabilidade.

A análise fatorial objetiva explicar as relações de correlação entre as variáveis estudadas, identificar fatores latentes e auxiliar a mensuração e validação de constructos complexos (ALMEIDA, 2013). Foi utilizado o método verossimilhança máxima, este método permite o cálculo de índices de ajustes que testam a significância das cargas fatoriais e as correlações entre fatores, e o cálculo de intervalos de confiança. Para determinar a quantidade de fatores extraídos foi utilizado o método eigenvalue, que indica que os fatores devem ter um autovalor maior que 1. Quanto aos métodos de rotação optou-se pela rotação obliqua com o método Oblimin. O resultado da extração da análise fatorial exploratória do QIE é apresentado no Quadro 3.

Quadro 3 - Análise fatorial exploratória - QIE

\begin{tabular}{|c|c|c|c|c|c|c|c|}
\hline \multirow[t]{2}{*}{ Fatores } & \multicolumn{3}{|c|}{ Autovalores iniciais } & \multicolumn{3}{|c|}{$\begin{array}{c}\text { Somas de extração de } \\
\text { carregamentos ao quadrado }\end{array}$} & \multirow{2}{*}{$\begin{array}{c}\begin{array}{c}\text { Somas de } \\
\text { rotação de } \\
\text { carregamentos } \\
\text { ao quadrado }\end{array} \\
\text { Total }\end{array}$} \\
\hline & Total & $\begin{array}{c}\text { \% de } \\
\text { variância }\end{array}$ & $\begin{array}{c}\% \\
\text { cumulativa }\end{array}$ & Total & $\begin{array}{c}\text { \% de } \\
\text { variância }\end{array}$ & $\begin{array}{c}\% \\
\text { cumulativa }\end{array}$ & \\
\hline 1 & 8,387 & 41,937 & 41,937 & 7,967 & 39,835 & 39,835 & 6,644 \\
\hline 2 & 2,315 & 11,577 & 53,513 & 1,803 & 9,014 & 48,849 & 2,890 \\
\hline 3 & 1,771 & 8,856 & 62,369 & 1,545 & 7,725 & 56,573 & 5,452 \\
\hline 4 & 1,084 & 5,421 & 67,789 &, 732 & 3,659 & 60,232 & 5,702 \\
\hline 5 & ,806 & 4,030 & 71,820 & & & & \\
\hline 6 &, 736 & 3,682 & 75,502 & & & & \\
\hline 7 & ,666 & 3,331 & 78,832 & & & & \\
\hline 8 &, 553 & 2,764 & 81,597 & & & & \\
\hline 9 & ,475 & 2,377 & 83,974 & & & & \\
\hline 10 & ,437 & 2,184 & 86,158 & & & & \\
\hline 11 & ,407 & 2,037 & 88,195 & & & & \\
\hline 12 & ,373 & 1,863 & 90,057 & & & & \\
\hline 13 & ,359 & 1,795 & 91,853 & & & & \\
\hline 14 & ,324 & 1,622 & 93,475 & & & & \\
\hline 15 & ,282 & 1,410 & 94,884 & & & & \\
\hline 16 & ,241 & 1,203 & 96,088 & & & & \\
\hline 17 & ,231 & 1,154 & 97,242 & & & & \\
\hline 18 & ,205 & 1,025 & 98,267 & & & & \\
\hline
\end{tabular}




\begin{tabular}{|l|r|r|r|}
\hline 19 &, 195 &, 975 & 99,242 \\
\hline 20 &, 152 &, 758 & 100,000 \\
\hline
\end{tabular}

Método de Extração: Máxima Verossimilhança.

${ }^{1}$ Quando os fatores são correlacionados, as somas de carregamentos ao quadrado não podem ser adicionadas para se obter uma variância total.

Fonte: elaborado pelos autores.

A análise fatorial exploratória do QIE forneceu 4 fatores que conjuntamente explicam, aproximadamente, $68 \%$ da variância, a partir do critério Kaiser, que prediz reter fatores com autovalor acima de 1.

Posteriormente foi realizada uma análise gráfica do número de fatores gerados que indicam a variância total explicada por cada fator, por meio do Gráfico de escarpa (Figura 3). O Gráfico escarpa mostra o número de componentes fatoriais extraídos em relação aos autovalores associados a esses fatores.

Figura 3 - Gráfico escarpa da análise fatorial exploratória - QIE

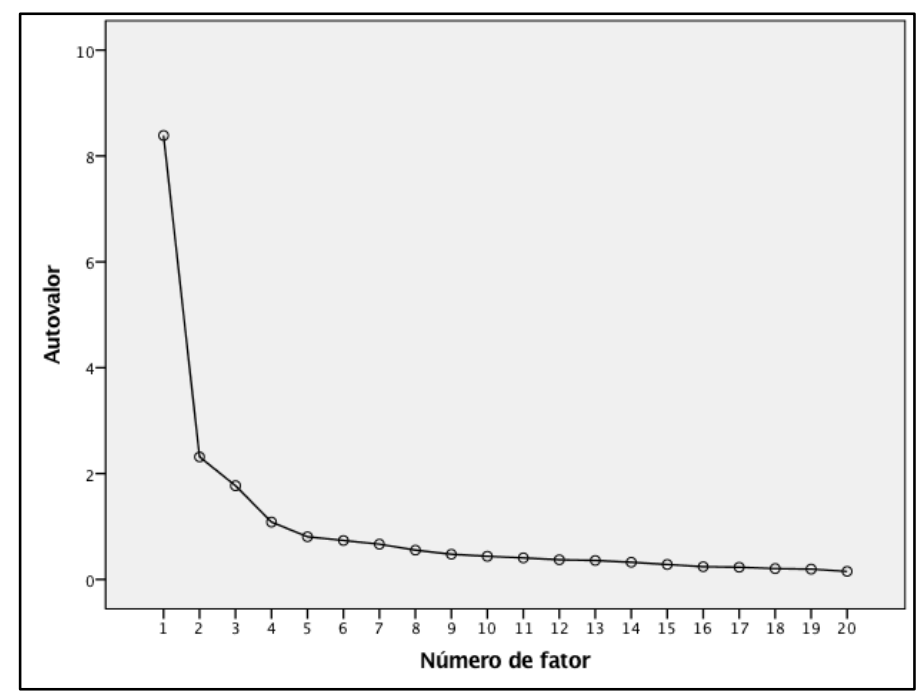

Fonte: elaborado pelos autores.

Para Almeida (2013) a rotação em uma análise fatorial exploratória tem como objetivo obter uma estrutura de correlação entre os itens de maneira que cada variável tenha altas cargas fatoriais nos itens que mensura, enquanto baixa correlação com os outros itens. A rotação dos fatores permite encontrar uma matriz de pesos facilmente interpretável. Optou-se pela rotação obliqua, que permite que os fatores estejam correlacionados, a carga fatorial e as correlações são distintas.

No Quadro 4 apresenta-se a matriz padrão dos fatores do QIE, que representa os pesos relativos de cada variável na composição do fator. Evidencia-se no respectivo quadro as comunalidades para as variáveis do instrumento e os itens marcados correspondem aos itens desenvolvidos para mensurar a atitude em relação ao empreendedorismo, apoio social, percepção de controle sobre o comportamento e intenção empreendedora.

Quadro 4 - Matriz padrão de fatores - QIE

\begin{tabular}{|l|l|l|l|}
\hline Variáveis & Fator & Comunalidades & Matriz de correlação de fator \\
\hline
\end{tabular}




\begin{tabular}{|c|c|c|c|c|c|c|c|c|c|c|}
\hline & 1 & 2 & 3 & 4 & Extração & Fator & 1 & 2 & 3 & 4 \\
\hline 1 & ,206 & ,252 &, 144 & ,397 &, 169 & 1 & 1,000 &, 245 & ,569 & ,637 \\
\hline 2 & ,528 & ,321 & ,365 &, 769 & ,596 & 2 &, 245 & 1,000 &, 210 & ,432 \\
\hline 3 & ,608 & ,370 & ,332 &, 816 & ,683 & 3 & ,569 & ,210 & 1,000 & ,397 \\
\hline 4 & ,618 & ,334 & ,387 & ,909 &, 833 & 4 & ,637 & ,432 & ,397 & 1,000 \\
\hline 5 & ,667 & ,269 & ,431 &, 768 & ,648 & & & & & \\
\hline 6 &, 188 & ,698 & , 181 & ,299 & ,488 & & & & & \\
\hline 7 &, 250 & ,926 & , 198 & ,383 & ,861 & & & & & \\
\hline 8 & ,264 &, 710 & ,194 & ,423 & ,521 & & & & & \\
\hline 9 & ,367 & ,298 & ,598 &, 345 & ,393 & & & & & \\
\hline 10 & ,501 &, 223 & ,727 & ,429 & ,553 & & & & & \\
\hline 11 &, 443 &, 154 &, 713 & ,301 & ,510 & & & & & \\
\hline 12 & ,405 &, 148 &, 824 &, 266 & ,686 & & & & & \\
\hline 13 & ,429 & ,066 & ,836 & 230 & ,717 & & & & & \\
\hline 14 & ,529 & , 159 & ,710 &, 356 & ,528 & & & & & \\
\hline 15 & ,679 & ,238 & ,552 & ,581 &, 535 & & & & & \\
\hline 16 & ,778 & , 189 & ,495 & ,567 & ,619 & & & & & \\
\hline 17 & ,696 & ,268 & ,446 & ,487 & ,497 & & & & & \\
\hline 18 & ,897 & ,211 & ,539 & ,534 & ,809 & & & & & \\
\hline 19 & ,791 & ,260 & ,446 & ,545 & ,631 & & & & & \\
\hline 20 & ,877 & ,252 & ,477 & ,579 & ,771 & & & & & \\
\hline
\end{tabular}

Método de Extração: Máxima Verossimilhança.

Método de Rotação: Oblimin com Normalização de Kaiser.

Fonte: elaborado pelos autores.

A partir da matriz padrão (Quadro 4), com rotação Oblimin, é possível distinguir claramente os fatores associados as dimensões do QIE de Liñán e Chen (2009). As comunalidades em sua maioria foram altas, excetuando-se as variáveis $1(0,169)$ e $9(0,393)$ que não foram bem explicadas pelos fatores extraídos.

Para mensurar a associação entre as dimensões do construto utilizou-se a análise de correlação (Quadro 5), que é uma medida do grau de relação linear entre duas variáveis. Optou-se pelo coeficiente de correlação de Pearson (r), que varia de -1 até +1 . Valores de coeficiente " $r$ " próximos a +1 indicam pouca dispersão, o que expressa uma correlação forte e positiva; já os valores próximos de "zero" indicam muita dispersão e ausência de relação entre as variáveis, e, por fim, o valor de "r" próximo de -1 significa pouca dispersão e uma correlação negativa forte (LOPES, 2003).

Para este estudo adotou-se a conversão de Dancey e Reidy (2013) que apontam para a seguinte classificação: $r=0,10$ até 0,39 (fraco); $r=0,40$ até 0,69 (moderado); $r=0,70$ até 1,00 (forte).

Quadro 5 - Correlação de Pearson para as dimensões do QIE

\begin{tabular}{|c|l|c|c|c|c|}
\cline { 3 - 5 } \multicolumn{2}{c|}{} & $\begin{array}{c}\text { Atitude } \\
\text { pessoal }\end{array}$ & $\begin{array}{c}\text { Normas } \\
\text { subjetivas }\end{array}$ & $\begin{array}{c}\text { Percepção } \\
\text { de controle }\end{array}$ & $\begin{array}{c}\text { Intenção } \\
\text { empreendedora }\end{array}$ \\
\hline \multirow{2}{*}{ Atitude pessoal } & Correlação de Pearson & $\mathbf{1}$ &, $396^{* *}$ &, $419^{* *}$ & $\mathbf{6 8 6}^{* *}$ \\
\cline { 2 - 6 } & Sig. (bilateral) & &, 000 &, 000 &, 000 \\
\hline \multirow{2}{*}{$\begin{array}{c}\text { Normas } \\
\text { subjetivas }\end{array}$} & Correlação de Pearson &, $396^{* *}$ & $\mathbf{1}$ &, $223^{* *}$ &, $311^{* * *}$ \\
\cline { 2 - 6 } $\begin{array}{c}\text { percepção } \\
\text { controle }\end{array}$ & Sig. (bilateral) &, 000 & &, 000 &, 000 \\
\cline { 2 - 6 } $\begin{array}{c}\text { Intenção } \\
\text { empreendedora }\end{array}$ & Sig. (bilateral) &, $419^{* *}$ &, $223^{* *}$ & $\mathbf{1}$ &, $583^{* *}$ \\
\cline { 2 - 6 } & Correlação de Pearson &, $686^{* *}$ &, 000 & &, 000 \\
\hline
\end{tabular}


** A correlação é significativa no nível 0,01 (bilateral).

Listwise $\mathrm{N}=401$

Elaborado pelos autores.

As dimensões de intenção empreendedora apresentaram correlação classificada como moderada (DANCEY; REIDY, 2013). Sendo a correlação mais elevada entre intenção empreendedora e atitude pessoal $(0,686)$ e a mais baixa entre percepção controle e normas subjetivas $(0,223)$. As correlações podem ser consideradas significativas pois apresentaram $\mathrm{p}$ valores inferiores a $10 \%(0,10)$.

Foram realizadas ainda análises de regressão para as variáveis do QIE. A regressão objetivou explicar a intenção empreendedora, utilizando como variáveis independentes as dimensões de atitude pessoal, normas subjetivas e percepção de controle. Inicialmente foram inseridos os preditores por meio do método stepwise no SPSS. Após a seleção dos preditores segundo os critérios do procedimento, foi realizada uma regressão com 1000 bootstraps para determinar intervalor de confiança para os parâmetros da regressão. No Quadro 6 apresenta-se a sumarização do modelo para a variável dependente intenção empreendedora.

Quadro 6 - Sumarização do modelo - intenção empreendedora ${ }^{\mathrm{c}}$

\begin{tabular}{|c|c|c|c|c|c|c|c|c|c|}
\hline \multirow{2}{*}{ Modelo } & \multirow{2}{*}{$\mathbf{R}$} & \multirow{2}{*}{$\mathbf{R}^{2}$} & \multirow{2}{*}{$\begin{array}{c}\mathbf{R}^{2} \\
\text { ajustado }\end{array}$} & \multirow{2}{*}{$\begin{array}{c}\text { Erro } \\
\text { padrão da } \\
\text { estimativa }\end{array}$} & \multirow{2}{*}{$\begin{array}{c}\text { Mudança } \\
\text { de R } \\
\text { quadrado }\end{array}$} & \multicolumn{3}{|c|}{$\begin{array}{c}\text { Estatísticas de } \\
\text { mudança }\end{array}$} & \multirow{2}{*}{$\begin{array}{c}\text { Sig. } \\
\text { mudança }\end{array}$} \\
\hline & & & & & & $\begin{array}{l}\text { Mudança } \\
\text { F }\end{array}$ & gl1 & gl2 & \\
\hline 1 &, $686^{\mathrm{a}}$ & ,471 & ,469 & 4,78337 & ,471 & 355,001 & 1 & 399 & ,000 \\
\hline 2 &, $759^{b}$ &, 576 &, 574 & 4,28459 & ,106 & 99,305 & 1 & 398 &, 000 \\
\hline
\end{tabular}

a. Preditores: (Constante), Atitude

b. Preditores: (Constante), Atitude, Percepção

c. Variável Dependente: Intenção empreendedora

Fonte: elaborado pelos autores.

A partir do Quadro 6 percebe-se que o coeficiente determinante $\left(\mathrm{R}^{2}\right)$ totalizou 0,574 , isso quer dizer que as variáveis independentes (Atitude e Percepção) conseguem explicar, em média, 57,4\% da variação da variável dependente (Intenção empreendedora). Observa-se que no modelo 1 (Quadro 6), que inclui somente a atitude como preditor, a mesma consegue explicar $47,1 \%$ da variância da intenção empreendedora. As dimensões de normas subjetivas e percepção de controle obtiveram resultados positivos, porém não tiveram o mesmo poder da atitude (Modelo 1 e 2). No Quadro 7 apresentam-se os coeficientes para a intenção empreendedora, por meio do boostrap com 1.000 observações.

Quadro 7 - Coeficientes da variável dependente intenção empreendedora ${ }^{\mathrm{a}}$

\begin{tabular}{|c|c|c|c|c|c|c|c|c|c|}
\hline & \multirow{2}{*}{ Modelo } & \multicolumn{2}{|c|}{$\begin{array}{c}\text { Coeficientes não } \\
\text { padronizados }\end{array}$} & \multirow{2}{*}{$\begin{array}{c}\text { Coeficientes } \\
\text { padronizados } \\
\text { Beta }\end{array}$} & \multirow{2}{*}{$\mathbf{t}$} & \multirow{2}{*}{ Sig. } & \multicolumn{2}{|c|}{ Colinearidade } & \multirow{2}{*}{$\begin{array}{l}\text { Condition } \\
\text { index }\end{array}$} \\
\hline & & B & $\begin{array}{c}\text { Erro } \\
\text { Padrão }\end{array}$ & & & & Tolerância & VIF & \\
\hline \multirow{2}{*}{1} & (Constante) &,- 454 &, 840 & &,- 541 &, 589 & & & 1,000 \\
\hline & Atitude & ,926 & ,049 & ,686 & 18,841 &, 000 & 1,000 & 1,000 & 6,885 \\
\hline \multirow{3}{*}{2} & (Constante) & $-3,498$ & ,812 & & $-4,309$ &, 000 & & & 1,000 \\
\hline & Atitude &, 724 & ,048 &, 536 & 14,926 &, 000 & 0,825 & 1,213 & 6,562 \\
\hline & Percepção & ,416 &, 042 & ,358 & 9,965 &, 000 & 0,825 & 1,213 & 8,374 \\
\hline
\end{tabular}


a. Variável Dependente: Intenção empreendedora

Fonte: elaborado pelos autores.

A partir do Quadro 7 é possível verificar que as variáveis Atitude e Percepção explicam significativamente a variável dependente intenção empreendedora, pois apresentaram significância menor que 0,05. A variável que apresenta maior poder de explicação da variável dependente é a Atitude, porque apresentou maior valor estatístico de $\mathrm{t}$ e maior valor de Beta padronizado, o que vem ao encontro da proposta de Ajzen e Fishein, onde atitude prediz e explica o comportamento humano (AJZEN; FISBHEIN, 2000). A colinearidade foi verificada por meio do indicador VIF, sendo que 1,000 para o modelo 1 , e de 1,213 para o modelo 2, ou seja, permaneceu abaixo do máximo aceitável para esta estatística, assim como o condition index máximo que foi de 8,374 o que indica que a multicolinearidade não foi um problema para esta regressão.

Verificou-se a regressão da variável atitude pessoal em relação as normas subjetivas e percepção de controle, porque para Liñán e Chen (2009) as duas dimensões determinam a atitude em relação ao comportamento empreendedor. Utilizou-se o mesmo método adotado para a variável intenção empreendedora, sendo o método stepwise e para os parâmetros de regressão utilizou-se o bootstrap com 1000 observações para uma maior confiabilidade (Quadro 8).

Quadro 8 - Sumarização do modelo - atitude pessoal ${ }^{\mathrm{c}}$

\begin{tabular}{|c|c|c|c|c|c|c|c|c|c|}
\hline \multirow{2}{*}{ Modelo } & \multirow{2}{*}{$\mathbf{R}$} & \multirow{2}{*}{$\mathbf{R}^{2}$} & \multirow{2}{*}{$\begin{array}{c}\mathbf{R}^{2} \\
\text { ajustado }\end{array}$} & \multirow{2}{*}{$\begin{array}{c}\text { Erro } \\
\text { padrão da } \\
\text { estimativa }\end{array}$} & \multirow{2}{*}{$\begin{array}{c}\text { Mudança } \\
\text { de R } \\
\text { quadrado }\end{array}$} & \multicolumn{3}{|c|}{$\begin{array}{c}\text { Estatísticas de } \\
\text { mudança }\end{array}$} & \multirow{2}{*}{$\begin{array}{c}\text { Sig. } \\
\text { mudança }\end{array}$} \\
\hline & & & & & & $\underset{\text { M }}{\text { Mudança }}$ & gl1 & gl2 & \\
\hline 1 &, $419^{\mathrm{a}}$ &, 175 & , 173 & 4,42431 & , 175 & 84,914 & 1 & 399 & ,000 \\
\hline 2 &, $521^{b}$ & ,272 & ,268 & 4,16310 & ,096 & 52,641 & 1 & 398 & ,000 \\
\hline
\end{tabular}

a. Preditores: (Constante), Percepção

b. Preditores: (Constante), Percepção, Normas

c. Variável Dependente: Atitude

Fonte: elaborado pelos autores.

A regressão foi significativa pois $o \mathrm{R}^{2}$ foi diferente de zero, o que indica que atitude pessoal pode se predita por normas subjetivas e percepção de controle. A regressão foi capaz de explicar 26,8\% da variação na atitude, sendo que a percepção de controle foi mais importante.

Quadro 9 - Coeficientes da variável dependente atitude pessoal ${ }^{\mathrm{a}}$

\begin{tabular}{|c|c|c|c|c|c|c|c|c|c|}
\hline & \multirow{2}{*}{ Modelo } & \multicolumn{2}{|c|}{$\begin{array}{c}\text { Coeficientes não } \\
\text { padronizados }\end{array}$} & \multirow{2}{*}{$\begin{array}{c}\begin{array}{c}\text { Coeficientes } \\
\text { padronizados }\end{array} \\
\text { Beta }\end{array}$} & \multirow[b]{2}{*}{$\mathbf{t}$} & \multirow[b]{2}{*}{ Sig. } & \multicolumn{2}{|c|}{ Colinearidade } & \multirow{2}{*}{$\begin{array}{l}\text { Condition } \\
\text { index }\end{array}$} \\
\hline & & B & $\begin{array}{c}\text { Erro } \\
\text { Padrão }\end{array}$ & & & & Tolerância & VIF & \\
\hline \multirow{2}{*}{1} & (Constante) & 10,866 &, 638 & & 17,036 &, 000 & & & 1,000 \\
\hline & Atitude &, 361 & ,039 & ,419 & 9,215 &, 000 & 1,000 & 1,000 & 5,595 \\
\hline \multirow{3}{*}{2} & (Constante) & 4,730 & 1,037 & & 4,562 &, 000 & & & 1,000 \\
\hline & Atitude &, 300 &, 038 & ,348 & 7,926 &, 000 & ,950 & 1,052 & 6,084 \\
\hline & Percepção & ,608 & ,084 & ,318 & 7,255 &, 000 & ,950 & 1,052 & 11,237 \\
\hline
\end{tabular}


a Variável dependente: atitude pessoal.

Fonte: elaborado pelos autores.

Os resíduos foram normais, não foram detectados problemas de colinearidade entre as variáveis, sendo $\mathrm{VIF}=1,000$ para o modelo 1 , e $\mathrm{VIF}=1,052$ para o modelo 2, o Condition Index totalizou em 5,595 para o modelo 1 e 11,237 para o modelo 2 (Quadro 9). Em ambas análises os resíduos estiveram normalmente distribuídos.

Diante da análise apresentada constata-se que a atitude pessoal pode ser predita por normas subjetivas e percepção de controle, e foi comprovado que a variável atitude pode explicar a intenção empreendedora, o que corrobora a $\mathrm{H}_{1}$.

Em seguida foi realizado o comparativo das variáveis conhecimento empreendedor e experiência prévia. Na Figura 4 apresenta-se a relação das dimensões do QIE diante dos indivíduos com conhecimento empreendedor e sem esse conhecimento.

Figura 4 - Conhecimento empreendedor e intenção empreendedora

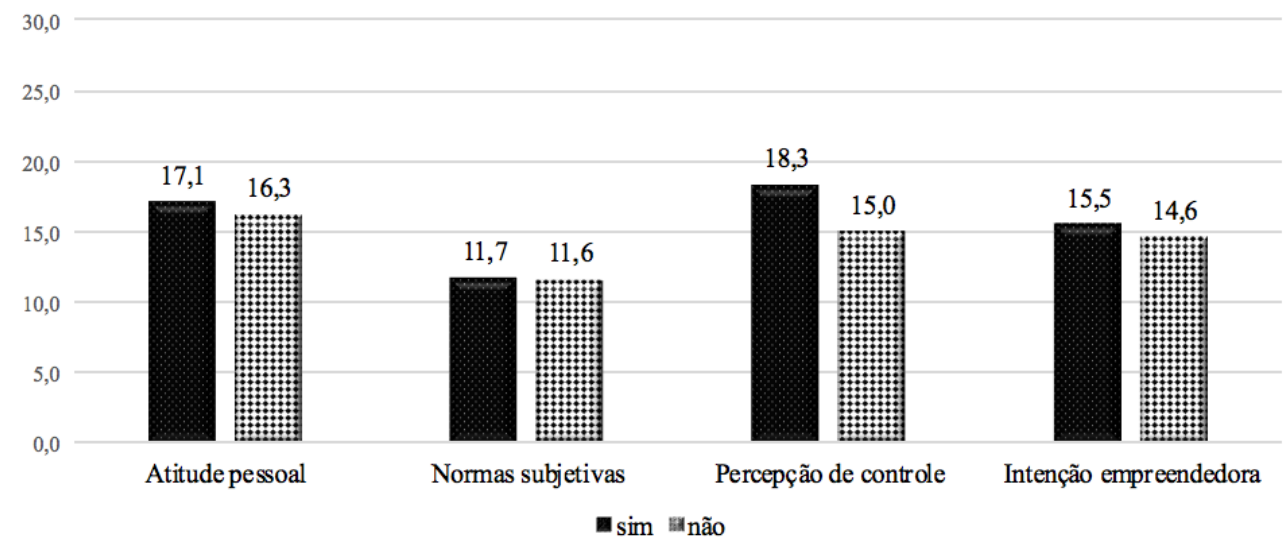

$1 \%$ dos pesquisados não respondeu esta questão.

Fonte: elaborado pelos autores.

Infere-se, a partir da Figura 4, que em todas dimensões do QIE os indivíduos com conhecimento empreendedor obtiveram pontuação maior diante dos indivíduos sem esse conhecimento, o que corrobora a $\mathrm{H}_{2 a}$ e reforça a importância de disciplinas de empreendedorismo em todos os níveis de ensino (KYRÖ, 2015). Salienta-se que a educação empreendedora pode desenvolver o potencial dos alunos para serem empreendedores em qualquer atividade que escolherem (DOLABELA; FILION, 2013).

Na Figura 5 apresenta-se a relação das dimensões do QIE diante dos indivíduos com experiência prévia e sem experiência.

Figura 5 - Experiência prévia e intenção empreendedora 


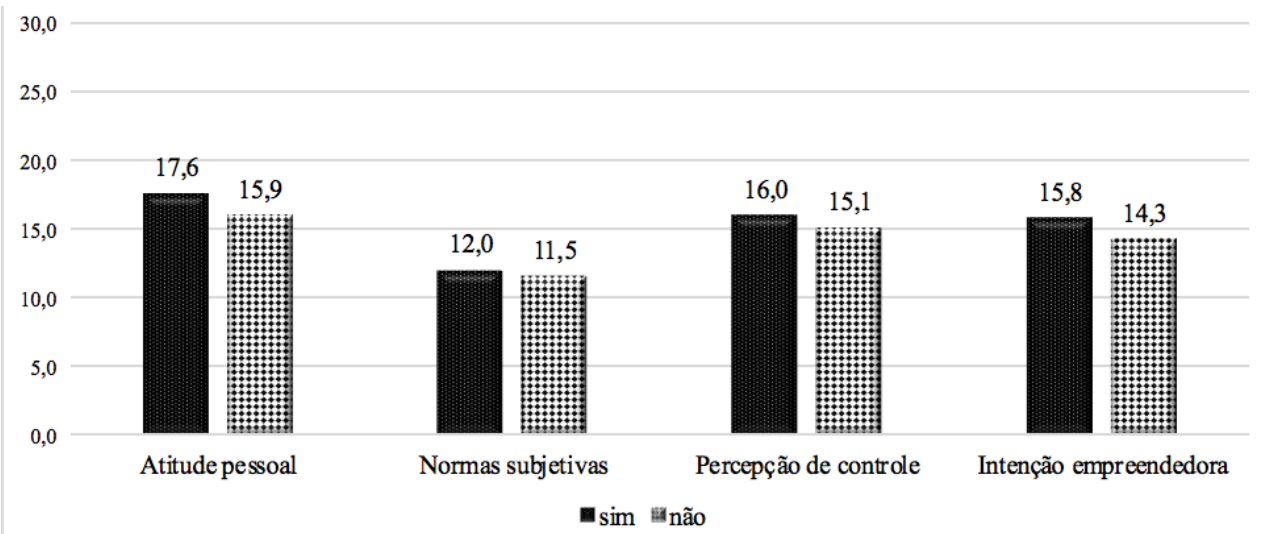

$1 \%$ dos pesquisados não respondeu esta questão.

Fonte: elaborado pelos autores.

A partir da Figura 5 constata-se que indivíduos com experiência prévia obtiveram pontuação superior diante dos indivíduos sem experiência, o que condiz com a $\mathrm{H}_{2 \mathrm{~b}}$. Isso corrobora com Dolabela (2008), quando, para o autor, aprende-se a ser empreendedor pela convivência com outros empreendedores, o comportamento empreendedor pode ser aprendido por meio da experiência de terceiros. Para Dolabela e Filion (2013), o ensino do empreendedorismo não deve restringir-se apenas ao âmbito acadêmico, este deve ser inserido com pequenos conceitos e práticas a partir da educação infantil, estimulando desde cedo aspectos empreendedores nos alunos, o que reforça a importância da prática do comportamento empreendedor desde cedo.

Nesse sentido, evidencia-se que discentes com conhecimento empreendedor e experiência prévia apresentaram pontuações superiores diante dos discentes sem conhecimento e experiência, o que vem de encontro a educação empreendedora, portanto, infere-se a existência de relação positiva entre educação empreendedora e intenção empreendedora, o que corrobora a $\mathrm{H} 2$.

Diante do apresentado, chama a atenção o percentual expressivo de alunos que nunca cursaram disciplinas relacionadas a empreendedorismo, 91\% (364 alunos), o que contrapõe o princípio da educação empreendedora, que para Clark (2006) fundamenta-se numa cultura empreendedora permeando por toda universidade. Deste modo, não se pode afirmar que a pontuação obtida na intenção empreendedora, do presente estudo, esteja diretamente relacionada a ações voltadas a educação empreendedora, pois poucos alunos, apenas 8\% (34 alunos), revelaram terem participado de disciplinas relacionadas ao tema.

\section{CONCLUSÕES}

Considerando-se que educação pode aumentar a qualidade e quantidade de estudantes empreendedores - inovadores, proativos e com iniciativa, tanto para atuarem em organizações quanto para tocarem seu próprio negócio - (LIMA et al., 2014), e o impacto do empreendedorismo no crescimento econômico e na geração de empregos (RAUCH; HULSINK, 2015), o presente estudo teve como objetivo analisar a intenção empreendedora de alunos de graduação de uma universidade diante da perspectiva da educação empreendedora.

Este objetivo foi plenamente contemplado, visto que, verificou-se que os discentes pesquisados possuem as quatro dimensões da intenção empreendedora. A partir da análise estatística infere-se que a atitude pessoal pode ser predita por normas subjetivas e percepção 
de controle, e foi averiguado que a variável atitude pode explicar a intenção empreendedora, o que condiz com a $\mathrm{H}_{1}$. Os resultados revelaram que os indivíduos com conhecimento empreendedor apresentaram níveis mais elevados de intenção empreendedora, o que é condizente com a $\mathrm{H}_{2 \mathrm{a}}$. Indivíduos com experiência prévia obtiveram pontuação maior em todas dimensões do QIE em relação aos indivíduos sem experiência, corroborando a $\mathrm{H}_{2 b}$, em que, existe relação positiva entre experiência profissional e níveis mais elevados de intenção empreendedora. Diante disso, constata-se a existência de relação positiva entre educação empreendedora e intenção empreendedora, corroborando a $\mathrm{H} 2$.

Nesse sentido, como contribuição acadêmica, os resultados do presente estudo podem contribuir para novas pesquisas relacionadas ao conhecimento empreendedor e experiência prévia, diante da intenção e educação empreendedoras, visto que apontam ligação entre o desenvolvimento de intenção empreendedora a partir da perspectiva da educação empreendedora. Além disso, os resultados também contribuem para o entendimento da intenção empreendedora em indivíduos com conhecimento empreendedor e experiência prévia e consolidam o uso do QIE (LIÑÁN; CHEN, 2009) e do modelo TCP (AJZEN, 1991) para analisar a intenção empreendedora dos indivíduos.

Como contribuição prática, os resultados revelam possíveis direções para o estímulo do empreendedorismo, principalmente sob a ótica de Pinto (2013), que entende que os empreendedores não são nascidos, mas desenvolvidos. Para isso, reforça-se a necessidade de incluir a educação empreendedora como pauta no ensino superior, inserindo-a como obrigatória junto aos currículos dos diferentes cursos de graduação, para que seu conteúdo alcance o estudante por meio de inserções teóricas e experiências práticas.

Este estudo apresenta algumas limitações, inicialmente existem outras variáveis que podem contribuir para explicar a intenção empreendedora de estudantes e que não foram contempladas pelo presente estudo, exemplifica-se: perfil de liderança (ATHAIDE, 2009), criatividade (HAMIDI; WENNBERG; BERGLUND, 2008), dentre outras. Quanto a amostra, uma amostra maior que contemplasse indivíduos em diversas regiões do país, com um maior percentual de indivíduos com conhecimento empreendedor e com experiência poderiam proporcionar maior variância dos dados e uma maior compreensão do fenômeno estudado. Para trabalhos futuros, sugere-se a inserção de uma metodologia qualitativa para melhor compreender os antecedentes da intenção empreendedora e como eles são percebidos pelos pesquisados. 


\section{REFERÊNCIAS}

AJZEN, I. The theory of planned behaviour. Organ. Behav. Hum. Decis. Process. 50, 179$211,1991$.

BAE, T. J.; QIAN, S.; MIAO, C.; FIET, J. O. The relationship between entrepreneurship education and entrepreneurial intentions: a meta-analytic review. Entrepreneurship: Theory and Practice, 38(2), 217-254, 2014.

BALCONI, S. B. A influência das atividades de educação empreendedora sobre as características empreendedoras dos alunos de graduação da UFSM. Dissertação (Mestrado em Gestão de Organizações Públicas). UFSM, Santa Maria, 2016.

BANDURA, A. Self-efficacy mechanism in human agency. American Psychology, 37, 122$147,1982$.

BARON, R. A. OB and Entrepreneurship: The reciprocal benefits of closer conceptual links. Em B. M. Staw \& R. M. Kramer (Eds.), Research in Organizational Behavior - An annual series of analytical essays and critical reviews (vol. 24, pp. 225-270). Oxford: Elsevier Science, 2002.

CLARK, B. Pursuing the entrepreneurial University. In: AUDY, J. L. N.; MOROSINI, M. C. (Org). Inovação e Empreendedorismo na Universidade. Porto Alegre: EDIPUCRS, 2006.

COPE, J. Toward a Dynamic Learning Perspective of Entrepreneurship. Entrepreneurship: theory and practice. Vol. 29, no 4, 2005.

COMISSAO EUROPEIA. Assessement of complience with the entrepreneurship education objective in the context of the 2006 spring council conclusions. Brussels, 2007.

COMISSAO EUROPEIA. Repensando educação: investindo em habilidades para melhores resultados socioeconômicos, 2012.

DOLABELA, F. Oficina do empreendedor. Rio de Janeiro: Sextante, 2008.

DOLABELA, F.; FILION, L. J. Fazendo revolução no Brasil: a introdução da pedagogia empreendedora nos estágios iniciais da educação. Revista de Empreendedorismo e Gestão de Pequenas Empresas, v. 3, n. 2, 2013.

FAYOLLE, A.; LINAN, F. The future of research on entrepreneurial intentions. J. Bus. Res. 67, 663-666, 2014.

GUERRA, M. J.; GRAZZIOTIN, Z. J. Educação empreendedora nas universidades brasileiras. In: LOPES, R. M. A. (Org.). Educação empreendedora: conceitos, modelos e práticas. Rio de Janeiro: Elsevier: São Paulo: SEBRAE, 2010.

KAUTONEN, T.; van GELDEREN, M.; FINK, M. Robustness of the theory of planned behav- iour in predicting entrepreneurial intentions and action. Enterp. Theory Pract. 39, 655- 
$674,2015$.

KYRÖ P. The conceptual contribution of education to research on entrepreneurship education. Entrepreneurship \& Regional Development 27(9-10): 599-618, 2015.

KUEHN, K.W. Entrepreneurial intentions research: implications for entrepreneurship education. J. Entrep. Educ. 11, 87-98, 2008.

KURATKO, D. F. Entrepreneurship: Theory, process, and practice. Cengage Learning. 2016.

KRUEGER, N. F.; REILLY, M. D.; CARSRUD, A. L. Competing models of entrepreneurial inten- tions. J. Bus. Ventur. 15, 411-432, 2000.

KRUEGER, N.F.; CARSRUD, A.L. Entrepreneurial intentions: applying the theory of planned behaviour. Entrep. Reg. Dev. 10, 315-330, 1993.

LANERO, A.; VÁZQUEZ, J. L.; GUTIÉRREZ, P.; GARCÍA, M. P. The impact of entrepreneurship education in European universities: an intention-based approach analyzed in the Spanish area. International Review on Public and Non-Profit Marketing, v. 8, n. 2, p. 111-130., 2011.

LOPES, C. L. J. Educação Empreendedora: Um estudo do projeto de empreendedorismo 10.0 aplicado aos alunos do curso técnico em informática. Revista de Empreendedorismo, inovação e tecnologia, 1(1)39-44, 2014.

LOPES, R. M. A. Educação Empreendedora: conceito, modelos e práticas. Rio de Janeiro: Elsevier, 2010.

LIMA, E.; NASSIF, V. M. J.; LOPES, R. M. A.; SILVA, D. Educação Superior em Empreendedorismo e Intenções Empreendedoras dos Estudantes - Relatório do Estudo GUESS Brasil 2013-2014. Grupo APOE - Grupo de Estudo sobre Administração de Pequenas Organizações e Empreendedorismo, PPGA-UNINOVE. Caderno de pesquisa, $n$. 3. São Paulo: Grupo APOE. 2014.

LIMA, E.; LOPES, R. M. A.; NASSIF, V. M. J.; SILVA, D. Ser seu Próprio Patrão? Aperfeiçando-se a educação superior em empreendedorismo. RAC, Rio de Janeiro, v. 19, n. 4, art. 1, pp. 419-439, Jul./Ago. 2015.

LIÑÁN, F.; CHEN, Y. W. Development and Cross-Cultural application of a specific instrument to measure entrepreneurial intentions. Entrepreneurship theory and practice, 33(3), 593-617, 2009.

MARESCH, D.; HARMS, R.; KAILER, N.; WIMMER-WURM, B. The impact of entrepreneurship education on the entrepreneurial intention of students in science and engineering versus business studies university programs. Technological forecasting and social change, 104, 172-179, 2016. 
MARTIN, B. C.; MCNALLY, J. J.; KAY, M. J. Examining the formation of human capital in entrepreneurship: a meta-analysis of entrepreneurship education outcomes. Journal of Business Venturing, 28(2), 211-224, 2013.

MCNALlY, J. J.; MARTIN, B. C.; HONIG, B.; BERGMANN, H.; PIPEROPOULOS, P. Toward rigor and parsimony: a primary validation of Kolvereid's (1996) entrepreneurial attitudes scales. Entrepreneurship \& Regional Development, 1-22. 2016.

MORAlES, J. F.; REBOlloso, E.; MOYA, M. Actitudes. Em J. F. Morales (Ed.), Psicología Social (pp. 495-621). Madrid, España: McGraw- Hill, 1994.

MORIANO, J. A.; PALACÍ, F. J.; MORALES, J. F. The psychosocial profile of the university entrepreneur. Psychology in Spain, 11(1), 72-84, 2007.

NECK, H. M.; GREENE, P. G. Entrepreneurship education: known worlds and new frontiers. Journal of Small Business Management, v. 49, n. 1, p. 55-70, 2011.

OLIVEIRA, B. M. D. F.; VIEIRA, D. A.; LAGUÍA, A.; MORIANO, J. A.; SALAZAR SOARES, V. J. Intenção empreendedora em estudantes universitários: adaptação e validação de uma escala (QIE). Avaliação Psicológica, 15(2), 187-196, 2016.

PAUL, J.; SHRIVATAVA, A. Do young managers in a developing country have stronger entrepreneurial intentions? Theory and debate. International Business Review, 2016.

PEREIRA, R. L. A. As competências do educador na difusão da cultura empreendedora: uma análise no ensino fundamental e superior de Belo Horizonte. 124 f. Dissertação (Mestrado Profissional em Administração) FIPEL - Fundação Cultural Dr. Pedro Leopoldo - Faculdades Integradas de Pedro Leopoldo, 2010.

PINTO, I. C. C. C. Rumo à universidade empreendedora: o potencial empreendedor dos alunos do ISEG. Dissertação (Marketing). School of Economics \& Management. Lisboa, 2013.

POLITIS, D. The process of entrepreneurial learning: a conceptual framework. Entrepreneurship Theory and Practice, p. 399-424, 2005.

RAUCH, A.J.; HULSINK, W. Putting entrepreneurship education where the intention to act lies. An Investigation into the Impact of Entrepreneurship Education on Entrepreneurial Behaviour, Academy of Management Learning \& Education. 2015.

ROCHA, E. L. C.; FREITAS, A. A. F. Avaliação do Ensino de Empreendedorismo entre Estudantes Universitários por meio do Perfil Empreendedor. Revista de Administração Contemporânea. V. 18, $\mathrm{n}^{\mathrm{o}}$ 4, Curitiba jul/ago, 2014.

SCHLAEGEL, C.; KOENIG, M. Determinants of entrepreneurial intent: a meta-analytic test and integration of competing models. Enterp. Theory Pract. 38, 291-332, 2014.

SEBRAE. Empretec: manual do participante. Brasília: Sebrae: 2011. 
SOUZA, E. C. L.; SOUZA, C. C. L.; ASSIS, S. A. G.; ZERBINI, T. Métodos e Técnicas de Ensino e Recursos Didáticos para o Ensino do Empreendedorismo em IES Brasileiras. In: SOUZA, E. C. L.; GUIMARÃES, T. A. (Orgs.) Empreendedorismo além do plano de negócio. São Paulo: Atlas: 2006.

SINGER, S.; AMORÓS, J. E.; ARREOLA, D. M. Global Entrepreneurship Monitor - 2014 Disponível em: <Global Report. http://www.gemconsortium.org/docs/download/3616>. Acesso 10 mar. 2016.

THOMPSON, E. R. Individual entrepreneurial intention: construct clarification and development of an internationally reliable metric. Enterp. Theory Pract. 33, 669-694, 2009.

TSENG, C. C. Linking Entrepreneurial Infrastructures and New Business Development: Entrepreneurship Development in Taiwan. The Journal of Entrepreneurship, v. 21, n. 1, p. 117-132, mar. 2012.

TUBBS, M; EKERBERG, S. The role of intentions of work motivations: Implications for goal-setting theory and research. Academy of Management Review, 16(1), 180-199, 1991.

ZAMPIER, M. A.; TAKAHASHI, A. R. W. Competências e aprendizagem empreendedora em MPE's educacionais. Revista Pensamento Contemporâneo em Administração. v. 8, n. 3, 2014. 